\title{
Bronquiolite aguda: (in)formar para prevenir
}

Margarida Cunha, ${ }^{1}$ Carolina Constant, ${ }^{2}$ Ana Sofia Mota, ${ }^{2}$ Teresa Bandeira ${ }^{2}$

\section{RESUMO}

Introdução: A bronquiolite aguda (BA) é uma infeção respiratória baixa frequente nos dois primeiros anos de vida. Os cuidados antecipatórios são eficazes na prevenção e deteção precoce de gravidade da doença.

Objetivos: Avaliar o conhecimento dos pais/cuidadores sobre BA e explorar determinantes desse conhecimento, incluindo fontes de informação e associação com medidas preventivas.

Métodos: Questionário a pais/cuidadores de crianças com idade inferior ou igual a 24 meses entre 1-15 de abril 2016. Caracterizaram-se os respondedores e os filhos e o conhecimento autopercecionado e real sobre BA, experiência anterior, fatores de risco, fontes de informação e conhecimento sobre medidas preventivas. O questionário foi aplicado por estudantes de medicina, em consulta programada, urgência hospitalar e em locais públicos. Análise descritiva e de associação (SPSS $21^{\circledR}$ ).

Resultados: Obtiveram-se 123 questionários completamente preenchidos. Os respondedores foram a mãe [92 (74,8\%)], o pai [23 (18,7\%)], outro [8 (6,5\%)], com idade média de 33,1 $\pm 7,9$ anos, ensino secundário/superior [100 (81,4\%)]. Dos participantes, $24(19,5 \%)$ eram fumadores e $56(46 \%)$ das crianças tinham pelo menos um irmão. O conhecimento real sobre BA [52 $(42 \%)]$ foi menor que o percecionado [89 $(72,4 \%)]$, verificando-se uma relação entre ambos $(p=0,03)$. O conhecimento real não é influenciado pelo número de irmãos $(p=0,539)$, habilitações literárias $(p=0,520)$, experiência prévia com BA $(p=0,059)$ ou fontes de informação [médico $(50,5 \%)$, folhetos $(5,6 \%)$ ] $(p=0,916)$, mas influencia o conhecimento sobre medidas preventivas [importância da lavagem das mãos $(p=0,001)$, risco da exposição tabágica durante a gravidez $(p=0,047)$ e após nascimento $(p=0,416)$, efeito protetor do aleitamento materno $(p=0,047)$ e maior risco de contágio em espaços fechados $(p=0,029)]$. Dos 34 pais/cuidadores com experiência prévia sobre BA, 13 (40,6\%) tinham recebido informação antes do diagnóstico.

Conclusão: Verificou-se que o conhecimento sobre BA influencia atitudes preventivas. A informação foi transmitida por médicos em cerca de metade dos casos. Estes resultados podem guiar a elaboração de campanhas mais eficazes.

Palavras-chave: Bronquiolite aguda; Prevenção; Fontes de informação; Conhecimento autopercecionado; Conhecimento real.

\section{INTRODUÇÃO}

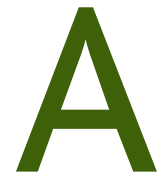

bronquiolite aguda (BA) é uma infeção aguda respiratória baixa com diagnóstico clínico, ${ }^{1-2}$ frequente nos dois primeiros anos de vida. ${ }^{1,3}$ Ocorre de forma epidémica e sazonal e evolui, na maioria dos casos, de forma autolimitada e benigna. ${ }^{1-3}$ Pela sua frequência e potencial gravidade é responsável por consumo significativo de cuidados de saúde. ${ }^{1-2}$ Fatores de risco para BA incluem os relacionados com a criança ${ }^{1-2,4-5}$ como idade inferior a três meses, prematuridade e doença crónica, socio-ambientais ${ }^{1-2,5}$ como a ausência de aleitamento materno, exposição a tabagismo parental (tabagismo durante a gravidez e a exposição ao fumo ambiental de tabaco) e número de irmãos ou frequência de creche. Medidas de controlo ambiental são eficazes na prevenção, ${ }^{1,3-6}$ o que, face à

1. Hospital de Santa Maria, Centro Hospitalar Lisboa Norte.

2. Faculdade de Medicina da Universidade de Lisboa. escassez de terapêuticas específicas, resulta num investimento com implicações na redução da morbilidade e do consumo de recursos de saúde, incluindo hospitalização. ${ }^{1,4}$

As medidas preconizadas na prevenção incluem que hábitos tabágicos devem ser ativamente inquiridos pelos profissionais de saúde e recomendadas medidas antitabágicas $^{1,3,5,7}$ e que o aleitamento materno deve ser incentivado, uma vez que se associa a menor gravidade e menor taxa de internamento ${ }^{1,5,7}$ por BA. Finalmente, a lavagem das mãos com sabão ou solução alcoólica, antes e depois do contacto com doentes infetados,,$^{1,3-5}$ deve ser fortemente recomendada ${ }^{1,5,7}$ com vista à redução da transmissão viral.

Em Portugal está previsto que familiares de crianças com idade inferior a dois anos recebam informação e treino através de um programa estruturado de promoção de saúde respiratória (norma de orientação clínica n. ${ }^{\circ} 016 / 2012$, da Direção-Geral da Saúde [DGS]). ${ }^{1}$ Este deve ser promovido 
pelos profissionais de saúde, que desempenham um papel essencial na transmissão de informação adequada e detalhada sobre medidas preventivas ${ }^{1}$ a serem transmitidas no momento do nascimento, antes da alta e durante as consultas de acompanhamento no primeiro ano de vida. ${ }^{5,7}$ Em relação à BA está prevista a transmissão de informação (manifestações e evolução, prevenção, tratamento e indicações para procurar o serviço de urgência) através da distribuição do folheto informativo ${ }^{1}$ disponível na norma da DGS. Desconhece-se, no entanto, o impacto da divulgação destas recomendações e da forma como o conhecimento de pais/cuidadores e experiências prévias influenciam a implementação de medidas preventivas. O objetivo deste estudo foi avaliar o conhecimento dos pais/cuidadores sobre BA, explorar determinantes desse conhecimento e a associação com a implementação de medidas preventivas.

\section{MÉTODOS}

Realizou-se um estudo transversal por aplicação de questionário, que abrangeu aspetos demográficos das crianças e pais, domínios sobre características relacionadas com a criança, circunstâncias do acompanhamento pediátrico, procura de informação, temas de pesquisa, comorbilidades e exposição ambiental ao fumo do tabaco. Construíram-se questões fechadas relacionadas com domínios da atualização de 2015 da Norma de Orientação Clínica n. ${ }^{\circ}$ 016/2012. Os itens foram adaptados aos objetivos e ajustados após revisão.

O questionário foi aplicado à amostra populacional urbana de conveniência constituída por pais/cuidadores de crianças com idade inferior ou igual a 24 meses, através de entrevista com suporte digital por médicos/estudantes de medicina, durante o mês de abril de 2016 (primeira quinzena), em ambiente de consulta programada (Unidade de Saúde Familiar [USF]), urgência hospitalar pediátrica de nível três e em locais públicos (centros comerciais, jardins e parques infantis). Foram identificados os respondedores acompanhados por crianças dentro da faixa etária.

O questionário incluiu 20 questões fechadas de escolha limitada (sim/não) ou múltipla, do tipo factual para descrição demográfica. Foi dada a hipótese de resposta não sabe/não responde. Para testar conhecimentos e atitudes dos pais/cuidadores sobre BA utilizaram-se questões de comportamento ou opinião e para avaliar as atitudes preventivas colocaram-se questões de hipóteses. As três questões abertas foram do tipo numérico e pretenderam caracterizar a idade do entrevistado e da criança e o número de filhos.
As questões relativas a contágio, transmissão e gravidade pretenderam testar o conhecimento sobre BA, que foi considerado positivo na presença de duas ou mais respostas corretas. Os determinantes do conhecimento explorados foram as habilitações literárias, o número de filhos, a forma de transmissão de informação e a experiência prévia com BA. ${ }^{8}$ As estratégias preventivas incluíram cinco questões relativas à lavagem das mãos, evicção do fumo do tabaco durante a gravidez e após o nascimento, aleitamento materno e evicção de ambientes sobrepovoados. Estas são medidas ambientais preventivas consideradas na literatura $\mathrm{a}^{1,3} \mathrm{e}$ difundidas à população através do folheto da norma DGS. ${ }^{1}$

Procedeu-se à análise descritiva de resultados, apresentada sob a forma de médias e percentagens. A relação entre o conhecimento real dos pais/cuidadores e seus determinantes foi feita através do teste de $\chi^{2}$. A relação entre o conhecimento real dos pais/cuidadores e o conhecimento de estratégias preventivas foi estimada a partir da média de respostas corretas, utilizando-se para isso o teste $t$ de Student. Consideraram-se resultados significativos valores de $p<0,05$, SPSS21 ${ }^{\circledR}$ (Statistical Package for Social Sciences, v. $21{ }^{\circledR)}$.

O estudo foi aprovado pela Comissão de Ética do Centro Hospitalar Lisboa Norte e do Centro Académico de Medicina de Lisboa.

\section{RESULTADOS}

Os 123 questionários realizados e obtidos presencialmente foram completamente preenchidos. Olocal de recolha foi registado em 80 (65\%). Destes, 30 (\%) foram aplicados nos cuidados de saúde primários (USF), 27 (\%) no serviço de urgência hospitalar e 22 (\%) em locais públicos.

\section{Demografia}

O Quadro I descreve as características demográficas dos pais/cuidadores. A idade média dos respondedores foi de 33,1 7,9, com idades mínimas e máximas de 17 e 64 anos respetivamente e com uma idade média materna e paterna de 33,1 anos. A maioria tinha o ensino secundário $(40,7 \%)$ ou superior $(40,7 \%)$. A mãe foi a respondedora em $92(74,8 \%)$ dos questionários e o pai em 23 (18,7\%). O pai/cuidador entrevistado é quem leva habitualmente a criança às consultas em $108(87,8 \%)$ dos casos e corresponde à mãe em 91 (84,3\%). Eram fumadores 24 (19,5\%) dos pais/cuidadores entrevistados, mas a exposição ao fumo de tabaco foi relatada em $58(47,1 \%)$ com a inclusão 


\begin{tabular}{|l|r|}
\hline \multicolumn{2}{|l|}{ QUADRO I. Caracterização demográfica de } \\
pais/cuidadores \\
\hline Parentesco $n$ (\%) & $92(74,8)$ \\
Mãe & $23(18,7)$ \\
Pai & $8(6,5)$ \\
Outro & $33,1 \pm 7,9$ \\
\hline Idade (anos), média \pm dp & {$[17 ; 64]$} \\
\hline [min; máx] & \\
\hline Quem costuma levar a criança a consulta & \\
$n$ (\%) & $107(86,9)$ \\
Mãe & $15(12,1)$ \\
Pai & $1(0,8)$ \\
Outro & \\
\hline Habilitações literárias $n(\%)$ & $50(40,7)$ \\
Ensino superior & $50(40,7)$ \\
Ensino secundário & $16(13)$ \\
$3^{\circ}$ ciclo & $7(5,7)$ \\
\hline $2^{\circ}$ e $1^{\circ}$ ciclo & $34(27,6)$ \\
\hline Exposição tabágica $n$ (\%) & $24(19,5)$ \\
Entrevistado fumador & $34(27,6)$ \\
Co-habitante fumador & \\
\hline Pais com filho com BA prévia $n(\%)$ & \\
\hline
\end{tabular}

QUADRO II. Caracterização demográfica das crianças

\begin{tabular}{l|r} 
Idade (meses), média $\pm \mathrm{dp}$ & $13 \pm 7,9$ \\
[min; máx] & {$[0,5 ; 24]$} \\
\hline Antecedentes pessoais $n(\%)$ & $109(88,6)$ \\
Saudável & $7(5,7)$ \\
Prematuro & $7(5,7)$ \\
Doença crónica & \\
\hline Número de irmãos & \\
$\geq 1$ irmão & $56(45,5)$
\end{tabular}

de co-habitantes fumadores. Foi referida experiência prévia com BA por $34(27,6 \%)$ dos pais/cuidadores.

A análise demográfica das crianças encontra-se no Quadro II. A idade média foi de $13 \pm 7,9$ meses, com um mínimo de 15 dias e máximo de 24 meses, a maioria era saudável [109 (88,6\%)] e 56 (45,5\%) tinham pelo menos um irmão.

\section{Conhecimento, atitudes e práticas}

Relativamente ao conhecimento percecionado pelos pais/cuidadores, 89 (72\%) afirmaram saber o que é BA.
Analisando as questões que estimam o conhecimento real sobre BA verificou-se que 57 (46\%) sabem que a BAé transmissível, 47 (38\%) que é contagiosa e 60 (49\%) respondem que nem sempre é uma doença grave. Responderam corretamente às três questões 25 (20\%) dos pais/cuidadores, a duas 27 (22\%), a uma 35 (29\%) e a nenhuma 36 (29\%). O conhecimento, traduzido pela resposta correta a duas ou mais das perguntas referidas, verificou-se em 52 (42\%) dos pais/cuidadores. Verificou-se uma relação entre o conhecimento real e o percecionado $(p=0,03)$.

Não se verificou correlação entre o conhecimento sobre BA, o número de irmãos $(p=0,539)$, as habilitações literárias $(p=0,520)$ ou a experiência prévia com $\mathrm{BA}(p=0,059)$.

$\mathrm{O}$ conhecimento dos pais/cuidadores sobre estratégias preventivas de BA foi avaliado através de cinco questões descritas no Quadro III. Para cada questão verificou-se uma taxa de resposta correta superior a $80 \%$. Responderam corretamente a todas as questões $79(64 \%)$, a quatro questões 27 (22\%), a três ou menos questões 17 (13,8\%). Nos pais/cuidadores em que se identificou conhecimento real sobre BA a média de respostas corretas foi superior $(p<0,001)$. Os pais/cuidadores com conhecimento real sobre BA identificam mais frequentemente a importância da lavagem das mãos ( $p=0,001)$, o risco da exposição tabágica durante a gravidez ( $p=0,047)$ e após o nascimento $(p=0,416)$, o efeito protetor do aleitamento materno $(p=0,047)$ e o maior risco de contágio em espaços fechados $(p=0,029)$ (Figura 1$)$.

Da análise da exposição tabágica verifica-se que mais de $90 \%$ dos pais/cuidadores a identificaram como um fator de risco para BA; contudo, destes, cerca de metade (48\%) afirmou ter hábitos tabágicos [19\% (21)] ou tinha cohabitantes fumadores [29\% (33)].

\section{Fontes de informação}

A informação sobre BA foi transmitida por: médico em 45 (50,5\%) dos casos [pediatra $(29,2 \%)$, médico de medicina geral e familiar $(21,3 \%)]$ e folhetos em $5(5,6 \%)$. A informação foi adquirida através de outras fontes em 32 (36\%) dos casos [formação/local de trabalho (12,4\%), Internet/TV $(12,4 \%)$, familiares não médicos $(11,2 \%)]$. Nos restantes $7,9 \%$ dos casos não foi especificada que outra fonte esteve na base da informação adquirida. Dos 34 pais/cuidadores com experiência prévia com $\mathrm{BA}$, a informação foi recebida antes do diagnóstico em 13 (40,6\%) dos casos. Não se verificaram diferenças entre as fontes de informação (médico versus outra fonte) e o conhecimento sobre BA $(p=0,916)$. 
QUADRO III. Avaliação do conhecimento sobre estratégias preventivas

\begin{tabular}{l|l|l} 
& Resposta correta & \multicolumn{1}{c}{$\boldsymbol{n ( \% )}$} \\
\hline $\begin{array}{l}\text { A lavagem das mãos é um método de prevenção eficaz } \\
\text { g exposição ao fumo do tabaco durante a gravidez aumenta o risco de infeção e de doença }\end{array}$ & Verdadeiro & $104(84,6)$ \\
\hline $\begin{array}{l}\text { A exposição ao fumo do tabaco após o nascimento aumenta o risco de infeção e de doença } \\
\text { grave }\end{array}$ & Verdadeiro & $108(87,8)$ \\
\hline $\begin{array}{l}\text { O aleitamento materno aumenta o risco de internamento por BA } \\
\text { Em quais destes locais há maior risco de contrair bronquiolite? }\end{array}$ & Falso & $113(91,9)$ \\
\hline
\end{tabular}

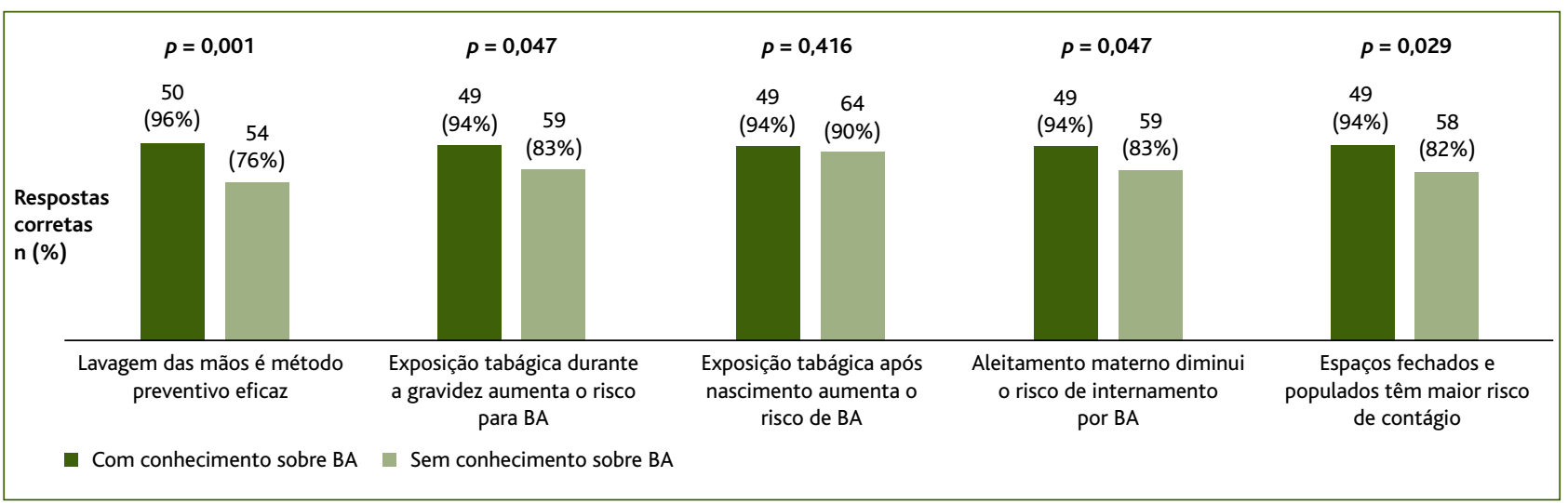

Figura 1. Relação entre conhecimento real sobre BA e conhecimento sobre medidas preventivas.

\section{DISCUSSÃO}

Tanto quanto se sabe, este é o primeiro estudo em Portugal que avalia os conhecimentos e fontes de informação de pais/cuidadores relativamente a atitudes e práticas relacionadas com BA.

Este estudo indica que é a mãe quem leva a criança à consulta médica em quase $90 \%$ dos casos. Resultados semelhantes encontraram-se num estudo realizado na Dinamarca, ${ }^{9}$ que conclui que as normas sociais podem ser influenciadoras. A confirmar-se, este achado pode ser indicador do alvo da literacia em saúde em temas relacionados com a criança. Por outro lado, com as alterações sociais que atribuem ao pai cada vez mais direitos para o exercício da parentalidade, poderá ser necessário desenvolver estratégias no sentido de capacitar ambos os pais para a gestão da saúde dos seus filhos.

Quanto às habilitações literárias, a amostra dividiu-se na sua maioria pelo ensino secundário $(40,7 \%)$ e superior $(40,7 \%)$, demonstrando valores superiores ao da popula- ção portuguesa registados nos Censos de 2011:32\% e 15\%, respetivamente, ${ }^{10}$ e mesmo na população ativa registada pelo INE em 2016: 26\% e 25,2\%. ${ }^{11}$ O nível elevado de educação da amostra, em relação ao da população portuguesa, poderá enviesar os resultados obtidos relativamente ao conhecimento sobre BA.

A prevalência de pais/mãe fumadores (19,5\%) é sobreponível aos da população portuguesa (cerca de 20\%), patentes no retrato do Inquérito Nacional de Saúde de 2014; ${ }^{12}$ contudo, a exposição tabágica dentro da família ascende a quase metade dos inquiridos. A exposição ao fumo ambiental do tabaco é nociva para todas as pessoas, nomeadamente para as crianças e grávidas. ${ }^{12}$ Nas crianças, o contacto físico próximo com os seus cuidadores aumenta também a exposição às toxinas do tabaco. ${ }^{13}$ Os efeitos nefastos do tabaco para a saúde da criança estão comprovados. ${ }^{13}$ A exposição tabágica pré e pós-natal conduz a uma diminuição dose-dependente da função respiratória, aumenta a morbilidade respiratória (aguda e crónica) 


\section{Dados Demográficos}

1. Idade da Criança (meses)

2. Entrevistado

$\square$ Pai $\square$ Mãe $\square$ Outro

3. Idade do Entrevistado

4. Habilitações Literárias

$\square$ 1. ${ }^{\circ}$ ciclo (até $4 .^{\circ}$ ano)

$\square$ 2..$^{\circ}$ ciclo (5..$^{\circ}$ e $6 .^{\circ}$ anos)

3. ${ }^{\circ}$ ciclo $\left(7 .^{\circ}, 8 .^{\circ}\right.$ e $9 .^{\circ}$ anos)

$\square$ Ensino secundário (10. ${ }^{\circ}, 11 .^{\circ}$ e $12 .^{\circ}$ anos)

$\square$ Ensino superior

5. Número de Filhos

6. Idade(s) do(s) outros filho(s)

(Especifique as idades, separadas por vígulas)

7. Comorbilidades

$\square$ Saudável

$\square$ Prematuro

$\square$ Doença crónica (cardíaca, respiratória, neurológica, outros)

8. Quem costuma levar o(s) seu(s) filho(s) às consultas médicas?

O próprio $\square$ Outro (quem)

9. É fumador(a)?

$\square \operatorname{Sim} \square$ Não

$\square$ Não, mas outro(s) fumador(es) habitam/convivem com o meu filho

\section{Conhecimento sobre a bonquiolite}

10. Sabe o que é o bronquiolite

$\square \operatorname{Sim} \square$ Não

11. Onde obteve essa informação?*

$\square$ Pediatra

Médico de família

Internet

Panfletos/Folhetos

Outra (qual)

12. Já teve um filho(a) com bronquiolite

$\square$ Sim

$\square$ Não
13. Se já teve um filho(a) com bronquiolite, obteve essa informação antes ou depois da infecção do seu filho(a)?

Antes $\square$ Depois

14. A bronquiolite é uma doença contagiosa?

$\square$ Verdadeiro

Falso

Não sabe/Não responde

15. A bronquiolite pode transmitir-se entre a criança e os pais/irmãos.

Verdadeiro

Falso

Não sabe/Não responde

\section{A prevenção da bronquiolite}

16. A lavagem das mãos é um método de prevenção eficaz.

$\square$ Verdadeiro

Falso

Não sabe/Não responde

17. A exposição ao fumo do tabaco durante a gravidez aumenta o risco de infecção e de doença grave.

$$
\text { Verdadeiro }
$$

Falso

Não sabe/Não responde

18. A exposição ao fumo do tabaco após o nascimento aumenta o risco de infecção.

$\square$ Verdadeiro

$\square$ Falso

$\square$ Não sabe/Não responde

19. O aleitamento materno aumenta o risco de internamento por bronquiolite.

$\square$ Verdadeiro

$\square$ Falso

$\square$ Não sabe/Não responde

20. Em quais destes locais há maior risco de contrair bronquiolite?

Fechados e populados (ex: centro comercial).

Amplos exteriores (ex: jardim).

Não sabe/Não responde

Email do inquirido, para divulgação do panfleto e do link da DGS, se os pais desejarem.

Figura 2. Questionário aplicado para avaliação do conhecimento sobre BA.

e a incidência de infeções respiratórias e de outras situações clínicas graves (sépsis, doença invasiva meningocó- cica, síndroma de morte súbita do lactente), incrementando o recurso a cuidados de saúde..$^{13}$ As admissões 
hospitalares por BA triplicam e a gravidade da doença aumenta. ${ }^{13-14}$

Apesar de a maioria dos respondedores afirmar possuir bom conhecimento sobre BA, os resultados sugerem que cerca de metade desconhece tratar-se de uma doença contagiosa e transmissível e mais de um terço considera que é sempre uma doença grave, de forma independente de experiência prévia, número de filhos ou grau de literacia. Está descrito que frequentemente as famílias não demonstram conhecimento suficiente na caracterização de sintomas ou nas medidas profiláticas a implementar. ${ }^{1}$

Já quanto ao conhecimento de estratégias preventivas relacionadas com BA, os resultados sugerem um maior conhecimento na amostra estudada, sendo estas identificadas corretamente por mais de $80 \%$ dos respondedores. Isto pode ser explicado pelo facto de serem medidas preventivas gerais, transmitidas de forma disseminada nos cuidados de saúde primários, a nível hospitalar e mesmo em campanhas publicitárias. No entanto, o conhecimento destas estratégias nem sempre modifica atitudes, como se verifica pela exposição tabágica que ocorre em quase metade da amostra, valor que não se altera significativamente dentro do grupo que sabe que esta aumenta o risco de infeção e de doença grave. Aliás, sabese que o conhecimento e as crenças em saúde são insuficientes para determinar mudanças de comportamento. ${ }^{8}$

A informação sobre BA foi transmitida por médico em apenas metade dos casos, fontes não médicas foram consultadas em cerca de $36 \%$ e o folheto em $5,6 \%$. Dos entrevistados que referiram já ter tido um filho com BA, a informação foi transmitida antes do diagnóstico em menos de metade. Tendo em conta estes resultados, a informação transmitida aos pais, quer sobre infeções respiratórias quer sobre BA, parece ser insuficiente e o folheto informativo da norma DGS pouco difundido. ${ }^{1}$

São limitações deste estudo a sua pequena dimensão, viés de nível de educação dos respondedores e amostra predominante com população urbana/residente em cidade. Em relação ao questionário deverá ser considerado o viés deste ser aplicado (por profissionais e estudantes) em vez de ser de autoaplicação e de uma das opções de resposta ser «Não sabe/Não responde». Igualmente no estudo realizado não foi avaliada a capacidade de caracterização de sintomas. Na realidade, um estudo com descrição de cenários e respostas qualitativas, por exemplo com utilização de uma escala, poderia ter permitido caracterizar melhor o conhecimento prático dos inquiridos.
No entanto, este estudo indica a necessidade de aumentar a disseminação de informação às famílias de crianças através dos profissionais de saúde. Outros estudos demonstraram que esta forma de veicular a informação é determinante da sua prática. ${ }^{8}$

\section{CONCLUSÃO}

O presente estudo suporta que, apesar do conhecimento percecionado pelos pais/cuidadores sobre BA, a doença respiratória baixa mais frequente em idade pediátrica, nem sempre se traduz em conceitos corretos (como, por exemplo, em relação à forma de transmissão e contágio da doença), mas parece influenciar positivamente a prática de atitudes preventivas.

Em Portugal, a informação a familiares de crianças com idade inferior a dois anos está integrada num programa estruturado de promoção de saúde respiratória, no qual os profissionais de saúde têm um papel-chave. O presente estudo demonstra que este exercício não se verifica em metade dos casos. Outros estudos deverão explorar as perceções dos profissionais de saúde sobre disseminação da informação, nomeadamente veiculada através de folhetos informativos oficiais, como é o caso da norma de orientação clínica n.o 016/2012, atualizada em 2015. Este estudo poderá incentivar a identificação de formas de intervenção no sentido de aumentar a literacia em saúde de pais/cuidadores.

Estudo elaborado no âmbito da disciplina de INTRODUÇÃO À MEDICINA DA CRIANÇA, do curso de mestrado integrado em medicina da Faculdade de Medicina da Universidade de Lisboa (coordenadora: Dra. Carolina Constant; regente: Prof. Doutora Teresa Bandeira).

\section{AGRADECIMENTOS}

Agradecimento aos estudantes da disciplina de IMC, ano letivo 2015/2016 e aos pais que colaboraram no projeto.

\section{REFERÊNCIAS BIBLIOGRÁFICAS}

1. Direção-Geral da Saúde. Diagnóstico e tratamento da bronquiolite aguda em idade pediátrica: norma n. ${ }^{\circ}$ 016/2012, de 19/12/2012, atualizada em 23/02/2015. Lisboa: DGS; 2015.

2. Scottish Intercollegiate Guidelines Network. Bronchiolitis in children: guideline no. 91. London: SIGN; 2006.

3. Piedra PA, Stark AR. Bronchiolitis in infants and children: treatment, outcome, and prevention. UpToDate [Internet]; 2016. Available from: https://www.uptodate.com/contents/bronchiolitis-in-infants-and-childrentreatment-outcome-and-prevention

4. Cincinnati Children's Hospital Medical Center. Evidence-based care guideline 
for management of bronchiolitis in infants one year of age or less with a first time episode. Cincinnati: Cincinnati Children's Hospital Medical Center; 2010.

5. Grupo de Trabajo de la Guía de Práctica Clínica sobre Bronquiolitis Aguda, Fundació Sant Joan de Déu, editor. Guía de práctica clínica sobre bronquiolitis aguda. Ministério de Sanidad y Politica Social; 2010. Available from: https://portal.guiasalud.es/wp-content/uploads/2018/12/GPC_475_ Bronquiolitis_AIAQS_compl.pdf

6. Baraldi E, Lanari M, Manzoni P, Rossi GA, Vandini S, Rimini A, et al. Inter-society consensus document on treatment and prevention of bronchiolitis in newborns and infants. Ital J Pediatr. 2014;40:65.

7. Ralston SL, Lieberthal AS, Meissner HC, Alverson BK, Baley JE, Gadomski AM, et al. Clinical practice guideline: the diagnosis, management, and prevention of bronchiolitis. Pediatrics. 2014;134(5):e1474-502.

8. Low MS, Tan H, Hartman M, Tam CC, Hoo C, Lim J, et al. Parental perceptions of childhood seasonal influenza vaccination in Singapore: a cross-sectional survey. Vaccine. 2017;35(45):6096-102.

9. Daly $M$, Groes F. Who takes the child to the doctor? Mom, pretty much all of the time. Appl Econ Lett. 2017;24(17):1267-76.

10. Instituto Nacional de Estatística. Censos 2011: resultados definitivos. Lisboa: INE; 2012.

11. PORDATA. População activa: total e por nível de escolaridade completo. PORDATA [Internet]; 2017. Available from: https://www.pordata.pt/Portugal/Popula\%C3\%A7\%C3\%A3o+activa+total+e+por+n\%C3\%ADvel+de+e scolaridade+completo-1008
12. Nunes E. Programa nacional para a prevenção e controlo do tabagismo 2012-2016. Lisboa: Direção-Geral da Saúde; 2013.

13. Espirito Santo R, Martins R, Pereira C, Salgado C, Sá G, Machado MC. Exposição tabágica e doença aguda na criança [Tobacco exposure and acute disease in children]. Acta Pediatr Port. 2017;48[1]:28-34. Portuguese

14. Jones LL, Hashim A, McKeever T, Cook DG, Britton J, Leonardi-Bee J. Parental and household smoking and the increased risk of bronchitis, bronchiolitis and other lower respiratory infections in infancy: systematic review and meta-analysis. Respir Res. 2011;12:5.

\section{CONFLITO DE INTERESSES}

Os autores declaram não ter quaisquer conflitos de interesse.

\section{FINANCIAMENTO}

O trabalho relatado neste manuscrito não foi objeto de qualquer tipo de financiamento externo (incluindo bolsas e investigação).

\section{ENDEREÇO PARA CORRESPONDÊNCIA \\ Margarida Cunha \\ E-mail: cunhapmargarida@gmail.com \\ http://orcid.org/0000-0002-0090-2464}

Recebido em 28-06-2018

Aceite para publicação em 22-08-2019

\section{ABSTRACT}

\section{ACUTE BRONCHIOLITIS: INFORM TO PREVENT}

Introduction: Acute bronchiolitis (AB) is the most common respiratory infection in the first two years of life. There is effective anticipatory care for prevention and severity identification. Aims of the study:To evaluate the knowledge of parents/caregivers about $A B$ and explore determinants of this knowledge, including sources of information and association with preventive measures.

Methods: Questionnaire to parents/caregivers of children aged less than or equal to 24 months between April 1-15, 2016. We characterized the responders and the children and the self-perceived and actual knowledge about $A B$, previous experience, risk factors, sources of information and knowledge about preventive measures. The questionnaire was applied by medical students, during the programmed or emergency appointment, and in public places. Descriptive and association analysis (SPSS $21^{\circledR}$ ) was applied.

Results: A total of 123 questionnaires were obtained. The respondents were the mother 92 (74.8\%), father 23 (18.7\%), other $8(6.5 \%)$, mean age $33.1 \pm 7.9$ years, secondary education $100(81.4 \%)$. Of the participants, $24(19.5 \%)$ were smokers and 56 (46\%) of the children had at least one sibling. The actual knowledge about AB [52 (42\%)] was lower than that self-perceived [89 $(72.4 \%)]$, with a relationship between both $(p=0.03)$. Real knowledge is not influenced by the number of siblings $(p=0.539)$, literacy $(p=0.520)$, previous experience with BA $(p=0.059)$ or sources of information [physician $(50,5 \%)$, leaflet $(5,6 \%)](p=0.916)$ but it influences knowledge about preventive measures [importance of hand washing $(p=0.001)$, risk of smoking exposure during pregnancy $(p=0.047)$ and after birth $(p=0.416)$, protective effect of breastfeeding $(p=0.047)$, and increased risk of contagion in enclosed spaces $(p=0.029)]$. Of the 34 parents/caregivers with prior AB experience, $13(40.6 \%)$ had received information before diagnosis.

Conclusion: We found that knowledge about $A B$ influences preventive attitudes. The information was transmitted by doctors in about $1 / 2$ of the cases. These results can guide the development of more effective campaigns.

Keywords: Acute bronchiolitis; Prevention; Information; Perceptional knowledge; Actual knowledge. 